\title{
Friction-induced vibration of a lubricated mechanical system
}

\author{
J-J. Sinou*, J. Cayer-Barrioz and H. Berro \\ Laboratoire de Tribologie et Dynamique des Systèmes, CNRS UMR 5513 \\ Ecole Centrale de Lyon, 36 avenue Guy de Collongue, 69134 Ecully cedex, France
}

\begin{abstract}
In a lubricated interface, the local dynamic responses can be complex and depend on molecular effects in the confined lubricating films. In a mechanical system comprising one or more of such interfaces, the influence of the local interfacial behaviour on the total vibrational response remains largely unknown. In this work, we propose a numerical model that incorporates realistic laws of local friction issued from previous experimental results. The objective is to characterize the dynamics of a lubricated system and to study its complex global responses triggered by the local interfacial behaviour. Both the stability analysis and vibrational oscillations of the mechanical system will be investigated through various operating conditions.
\end{abstract}

Keywords

lubrication - dynamics - stability - mechanical system

\section{Introduction}

Nowadays, one of the important challenges in mechanical design is the ability to predict and reduce structural vibrations. These vibrations can be fed by external sources or by themselves, such as friction-induced vibrations. In all cases, these vibrations are hardly controllable and result in elevated stresses, exceeding those expected over nominal operation cycles. On one hand, the encountered material fatigue can significantly reduce the lifetime of the mechanical components while on the other hand the acoustic emissions from such vibrations may be a source of discomfort, for example, brake-squealing noise in automobile.

Several mechanisms have been proposed in the literature to explain friction-induced vibrations. In self-sustained systems, these mechanisms fall into two distinct categories. First, one can mention the instabilities that depend mainly on geometrical characteristics of the system, such as sprag-slip or modal coupling through friction [14]. Sprag-slip instabilities were first described by Spurr [5]: the instability of the stationary position arises from a kinematic coupling between the variation of normal and friction forces. For modal coupling through friction instabilities, Jarvis and Mills [6] first showed that the increase in friction induces a displacement of the two natural frequencies of the system: respectively in normal and tangential directions towards each other, until they become equal at the so-called Hopf bifurcation point. The static equilibrium position then becomes highly unstable, even for infinitesimal perturbations. These instabilities result in oscillations. In the second category as previously referred, one can find stick-slip instabilities that may occur with a variation of the friction coefficient. For instance, discontinuous static/dynamic friction transition or a decrease in dynamic friction with an increase in velocity will generate saw-tooth like vibrations that result from stick and slip states. On the other hand, in externally excited systems such as those involving a varying normal force or varying sliding velocity, the system can become unstable when a delay occurs between the external 
excitation and the system frictional response, a phenomenon commonly detected in experiments.

Friction-induced vibrations are in reality the consequence of a combination of those mechanisms, weighted relatively to the system configuration, operating and environmental conditions. The problem can become more complicated when lubricated interfaces are involved. In the boundary lubrication regime, a thin fluid film is confined between the two shearing surfaces. It displays specific properties that strongly differ from those of the bulk. These changes have been extensively studied, thanks to the development of the Surface Force Apparatus (SFA) and Atomic Force Microscopy (AFM) techniques [7-9] and are usually related to confined film relaxation times, orders of magnitude larger than those of the bulk [10], and to liquid/solid transitions [11-12] within the interfacial layer. Evidence of layering may be observed [11, 13-15] although liquid/solid transitions may also occur without any ordering [14, 16-17]. The friction response of such confined layers under shear will depend on the operating conditions (contact pressure, sliding velocity, and temperature), the nature of the surfaces and their mechanical and topographic characteristics, and the nature and shape of the confined fluid molecules [18-19]. In the case of polymer and selfassembled monolayers [20-22], shear experiments at the nanoscale also show long relaxation times, characteristic memory length and link friction dynamics to molecular organization.

In order to account for these effects, various models have been developed to describe the frictional response of dry/lubricated single/multi-asperity contacts [23-30]. For instance, the so-called phenomenological 'state and rate' approach assumes that the interfacial area is large enough to self-average and allows one to model the collective dependence of friction on both the internal degrees of freedom of the interfacial layer and the characteristics of the shear motion. Indeed, the dissipative pinning/depinning of mesoscopic domains governs the interfacial rheology and the frictional response of contacts. Therefore, considering the interface as statistically interacting units is a promising way of modelling the friction.

In this manuscript, the impact of local interfacial friction dynamics on the overall system behaviour is investigated through numerical modelling that relies on realistic experimental results for lubricated friction. The interest of this work emerges from the lack in literature of studies focused on friction-induced vibrations with lubricated interfaces. Besides, we will highlight the following paradox: although lubricated systems are supposed to reduce the frictional losses and improve the lifetime of mechanical components, they can generate undesirable vibrations leading to contact deterioration and material fatigue when badly designed.

This manuscript is organized as follows. The model is first described including the equations of motion and details on the considered lubricated friction law. In the later sections, the stability of the self-excited system is discussed over its possible operating regimes and vibrational responses cycles are investigated in the presence of variable external excitations including alternative sliding.

\section{Model description}

In this section, the single degree-of-freedom block on a moving belt model is described. Then, the lubricated friction model that is based on SFA experimental results by Mazuyer et al [19] is presented.

\subsection{Mechanical system}

The mechanical system under study, shown in Figure 1, is composed of a mass held against a moving band with a confined lubricated interface. For the sake of simplicity, 
we assume that the mass and band surfaces are always in contact. This assumption may be due to a preload applied to the system. The equation of motion for the system is defined as follows:

$$
m \ddot{x}+c \dot{x}+k x=F_{t}\left(v_{r e l}\right)
$$

where $x(t)$ is the displacements. $m, c$ and $k$ stand respectively for mass, damping and stiffness of the system. $v_{\text {rel }}$ defines the relative velocity of the mass given by $v_{\text {rel }}=v_{\text {band }}-\dot{x} . F_{t}\left(v_{\text {rel }}\right)$ represents the tangential friction force:

$$
F_{t}\left(v_{r e l}\right)=\mu\left(N, v_{r e l}\right) N
$$

where $N$ defines the constant normal force and $\mu\left(N, v_{\text {rel }}\right)$ the friction coefficient.

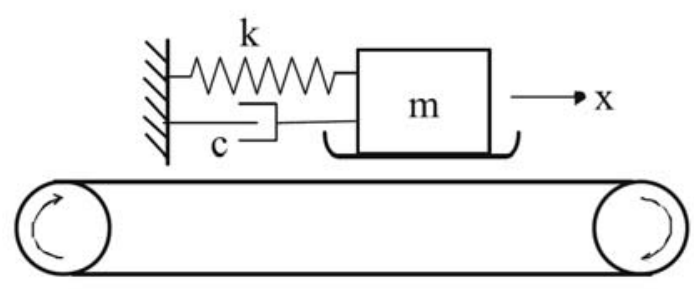

Figure 1: Mechanical system considered in this work

\subsection{Lubricated friction model}

Various models have been proposed in the literature [24-29, 31-32]. However, their main drawback relies on the lack of physical meaning for the variables used in the equations. In this framework, we will consider a general friction model based on the kinetics of formation and rupture of adhesive bonds between two shearing surfaces with an additional viscous contribution. This model, originally derived from a theory of adhesive friction between elastomeric surfaces [23,33], has been successfully applied to predict new instability regimes [30] and velocity-dependent friction response of surfactant monolayers [19,34] and of more complex adsorbed polymer layers [22].

The confined lubricated interface, considered as a viscoelastic medium, has a shear elastic modulus, $G$, and a viscosity, $\eta$. The total contact area, A, can be described as schematically depicted in Figure 2: at any time of shear, it consists of $N_{j}$ independent adhesive nanodomains, referred to as junctions, of average individual area, $\delta A$. During shear, individual junctions are continuously formed and broken incoherently. Each junction can either be in a bonded state and it contributes elastically to friction or in a free state and then it participates to friction dissipation with a viscous contribution. The junction activation involves two characteristic times: $\tau_{0}$, the mean time to break a junction due to thermal fluctuations under zero shear force, and $\tau$, the mean time to activate or reactivate a junction thermally. A junction is assumed to remain in the bonded state until it is elastically stretched under shear, up to a yield distance I $^{*}$ beyond which it becomes depinned or free. Figure $3 a$ and $b$ respectively present a schematic of a bonded junction respectively at rest and stretched while Figure $3 c$ illustrates $a$ free junction. The mean lifetime of $a$ bonded junction, $\left\langle t_{b}\right\rangle$, can be calculated from its survival probability, such as:

$$
\left\langle t_{b}\right\rangle=\tau_{0}\left(1-e^{-\frac{v_{0}}{v_{r e l}}}\right)
$$

with $v_{0}=I^{*} / \tau_{0}$ is the critical sliding velocity at the junction becomes free under shear. According to this model, for a constant normal force $N$, the friction coefficient $\mu$ results from two terms elastic and viscous contributions, as follows: 


$$
\mu\left(N, v_{r e l}\right)=\operatorname{sign}\left(v_{r e l}\right)\left(\varphi \frac{A G I^{*}}{D N} \frac{v_{r e l}}{v_{0}}\left(\frac{1-\left(1+\frac{v_{0}}{v_{r e l}}\right) e^{-\frac{v_{0}}{v_{r e l}}}}{1-e^{-\frac{v_{0}}{v_{r e l}}}}\right)+(1-\varphi) \frac{A}{d N} \eta v_{r e l}\right)
$$

where $\mathrm{D}$ is the lubricated interface thickness,; $\mathrm{d}$ is the thickness of the interpenetration zone between adsorbed layers, as defined in Figure $3 a, \varphi=\frac{\left\langle t_{b}\right\rangle}{\left\langle t_{b}\right\rangle+\tau}$ is the ratio between the number of junctions at $v_{\text {rel }}$ and that at rest, $\tau=\tau_{r e f}\left(\frac{v_{r e l}}{v_{r e f}}\right)^{k_{1}}$ is the mean time to activate or reactivate a junction thermally and is given by decreasing power law with a reference time, $\tau_{\text {ref, }}$ a reference velocity, $v_{\text {ref, }}$ and a power index, $k_{1}$, ranging from -1 to 0 , and the viscosity $\eta=\eta_{\text {ref }}\left(\frac{v_{r e l}}{v_{r e f}}\right)^{k_{2}}$ is the dynamic viscosity of the shear-thinning lubricant. $\eta_{\text {ref }}$ is a reference viscosity and $k_{2}$, a power index varying between -1 and 0 , is dependent on the nature of the lubricant. It is noteworthy that the contact area $\mathrm{A}$ also depends on the normal force $\mathrm{N}$ according to Jonhson-KendallRoberts adhesive contact law [35].

In the remaining part of this paper, the contact is lubricated with a blend composed of $0.5 \% \mathrm{w} / \mathrm{w} \mathrm{N}$-alkyl dioelate diamine, a friction modifier, dispersed in polyalphaolefin base oil. The viscosity of the lubricant at ambient pressure and temperature, measured using a capillary viscometer, is $18.10^{-3}$ Pa.s. Friction experiments on adsorbed diamine monolayers were performed on the molecular tribometer of the Ecole Centrale de Lyon [19, 34]. The results are described in detail in [19]. Under a constant normal force of $10^{-3} \mathrm{~N}$ and for a thickness $\mathrm{D}$ of $4.2510^{-9} \mathrm{~m}$, i.e. twice the length of the diamine molecule, the contact area $A$ is $28.310^{-12} \mathrm{~m}^{2}$. Dynamic measurements carried out at $37 \mathrm{~Hz}$ allow us to estimate the shear elastic modulus of the confined lubricated interface, $\mathrm{G}=3.910^{6} \mathrm{~Pa}$. The evolution of the friction coefficient as a function of the sliding velocity is presented in Figure 4 . Three friction regimes can be detected: for a sliding velocity lower than $4.110^{-12} \mathrm{~m} / \mathrm{s}$, the friction is rather high at around 0.1. This large value mainly emerges from the elastic contribution arising from the bonded junctions. It corresponds to a static friction. As the sliding velocity increases, the number of free junctions goes up and the friction decreases. This regime lasts until the velocity reaches $1.3510^{-7} \mathrm{~m} / \mathrm{s}$. Above this velocity, the friction response becomes purely viscous, due to a large number of free junctions and the friction increases linearly with the sliding velocity.

The application of the friction model - Equation (4) - to experimental data gives:

$\mathrm{v}_{0}=1.610^{-12} \mathrm{~m} / \mathrm{s}, \mathrm{v}_{\text {ref }}=10^{-10} \mathrm{~m} / \mathrm{s}, \mathrm{I}^{*}=1.610^{-9} \mathrm{~m}, \mathrm{~d}=10^{-10} \mathrm{~m}, \tau_{0}=1000 \mathrm{~s}, \tau_{\text {ref }}=18$ $\mathrm{s}, \mathrm{k}_{1}=-0.41, \mathrm{k}_{2}=-0.62$ and $\eta_{\mathrm{ref}}=900$ Pa.s. The physical meaning of these values is discussed in detail in [19]. In the following expression of the friction coefficient $\mu\left(N, v_{\text {rel }}\right)$ will be denoted by $\mu\left(v_{\text {rel }}\right)$ due to the fact that the normal force $N$ is constant. 


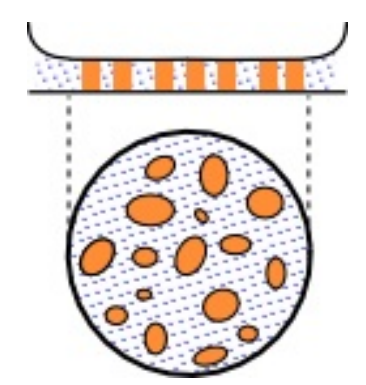

Figure 2: Schematic of the contact area A that is composed of bonded and free junctions

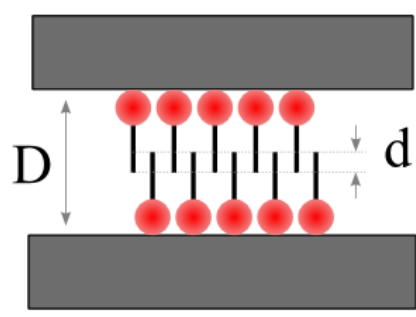

(a)

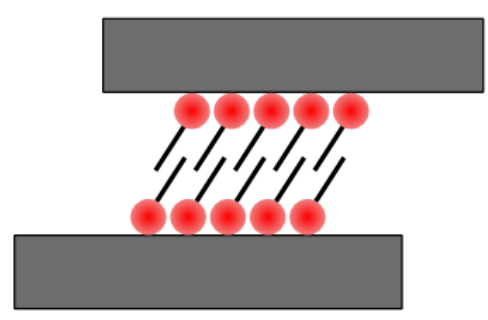

(b)

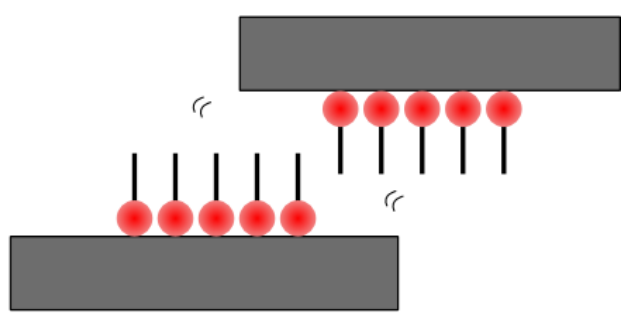

(c)

Figure 3: Shear-induced rupture process of junctions a) a bonded junction with an interpenetration zone of thickness $d, b$ ) an elastically stretched bonded junction, c) a free junction

\subsection{Complete mechanical system with the lubricated friction model}

Finally, by introducing the constants $\zeta=\mathrm{c} /(2 \sqrt{\mathrm{km}}), \omega_{0}=\sqrt{\mathrm{k} / \mathrm{m}}$ and the hyper-time $\mathrm{t}^{\prime}=\omega_{0} \mathrm{t}$, Equation (1) becomes:

$$
\ddot{x}+2 \zeta \dot{x}+x=\frac{\mu N}{k}
$$

with $\dot{x}=\frac{\partial x}{\partial t^{\prime}}$ and $\ddot{x}=\frac{\partial^{2} x}{\partial t^{\prime 2}}$. The expression of the friction coefficient $\mu$ is given in Equation (4). Numerical values of the parameters are $\mathrm{m}=0.1 \mathrm{~kg}, f_{0}=\frac{\omega_{0}}{2 \pi}=10 \mathrm{~Hz}$, $\zeta=0.01$ and $\mathrm{N}=10^{-3} \mathrm{~N}$.

\section{Numerical study}

In this part of the paper, the stability analysis and the nonlinear behaviour of the system with a confined lubricated interface for different operating conditions will be investigated.

\subsection{Stability analysis}

The stability analysis of the static sliding equilibrium point is the first step while studying nonlinear systems subjected to instability phenomena [2]. For a given set of parameters, a static equilibrium position can become unstable and nonlinear oscillations occur.

This analysis is performed in two steps [36]. The first step in the solution procedure is to obtain the steady state operating point, called the equilibrium point, for the nonlinear equation defined in Equation (5). This equilibrium point $x_{0}$ is calculated by 
solving the nonlinear static equations for a given normal force. It satisfies the following conditions:

$$
x_{0}=\frac{\mu(\dot{x}=0) N}{k}=\frac{\mu\left(v_{r e l}=v_{\text {band }}\right) N}{k}
$$

It is observed that the equilibrium point depends on the ground sliding velocity $v_{\text {band }}$ via the friction coefficient.

Secondly, the stability is investigated on the linearized equations by assuming small perturbations $\bar{x}$ around the equilibrium point $x_{0}$ i.e. $x=x_{0}+\bar{x}$. The linearization of the equation of motion for a small displacement from the equilibrium position gives the following differential equation:

$$
\ddot{\bar{x}}+\left(2 \zeta+\frac{\omega_{0} \mu_{0}^{\prime} N}{k}\right) \dot{\bar{x}}+\bar{x}=0
$$

The term $\mu_{0}^{\prime}$ corresponds to the linearized nonlinear forces which is composed by the following Jacobian value for each operating condition:

$$
\mu_{0}^{\prime}=\frac{\partial \mu}{\partial v_{\text {rel }}}\left(v_{\text {band }}\right)
$$

We consider that the normal load $N$ and the ground sliding velocity $v_{\text {band }}$ are both constant with time. In this case, the system is said to be self-sustained since all possible vibrations would occur at the natural frequency of the system.

Figure 4 also shows the calculated Jacobian values corresponding to the friction coefficient $\mu$ previously defined in Equation (4) taking into account the elastic and viscous contributions.

Finally, the stability of the equilibrium point is evaluated by considering the RouthHurwitz criterion [37] given by:

$$
\zeta+\frac{\omega_{0} \mu_{0}^{\prime} N}{2 k}>0
$$

After calculation, the complete expression giving the stability condition of the equilibrium point is:

$$
c>\frac{\partial F_{t}(\dot{x}=0)}{\partial \dot{x}}=\frac{-\varphi A G \tau_{0}}{D}\left(\frac{1-\left(1+\frac{v_{0}}{v_{r e l}}\right) e^{-\frac{v_{0}}{v_{r e l}}}}{1-e^{-\frac{v_{0}}{v_{r e l}}}}-\frac{\left(\frac{v_{0}}{v_{r e l}}\right)^{2} e^{-\frac{v_{0}}{v_{r e l}}}}{\left(1-e^{-\frac{v_{0}}{v_{r e l}}}\right)^{2}}\right)-\frac{(1-\varphi) A \eta}{d}
$$

Obviously, the stability of the equilibrium position depends on the ground velocity. If the ground velocity lies in zone 1 or 3 of the friction curve, as indicated in Figure 4, then the Jacobian value $\mu_{0}^{\prime}$ is positive and the equilibrium position is stable. However, in zone 2 where the friction $\mu\left(v_{\text {band }}\right)$ is a decreasing function of the relative velocity, a minimum value of $\zeta$ i.e. the non dimensional structural damping $\zeta=\mathrm{c} /(2 \sqrt{\mathrm{km}})$, is required to avoid instability.

Consequently, three possible stability scenarios can be encountered as a function of the ground velocity as shown in Figure 5. First, if the slope of the friction coefficient with the relative velocity is positive (i.e. $v_{r e l}<4.10^{-12} \mathrm{~ms}^{-1}$ ), then the system is naturally stable. Second, for a range of velocities between $4.10^{-12} \mathrm{~ms}^{-1}$ and $1.3510^{-7}$ $\mathrm{ms}^{-1}$, the system is by nature unstable in the absence of structural damping. However, 
the mechanical system can be stabilized by adding an appropriate non dimensional structural damping $\zeta$ for the range of ground velocity between $3.10^{-8} \mathrm{~ms}^{-1}$ and $1.3510^{-}$ ${ }^{7} \mathrm{~ms}^{-1}$ : increasing the quantity of $\zeta$ increases the stability of the equilibrium point if all the other parameters are kept constant, as indicated in Figure 5. However, for a ground velocity value between $4.10^{-12} \mathrm{~ms}^{-1}$ and $3.10^{-8} \mathrm{~ms}^{-1}$, it can be observed that the mechanical system with the lubricated friction model is unstable whatever the value of the non dimensional structural damping $\zeta$. For example, a numerical simulation performed with a ground velocity of $2.10^{-8} \mathrm{~ms}^{-1}$ results in a stick-slip motion as shown in Figure 6: the mass of the mechanical system sticks until the tension force reaches the value of the friction force at the lubricated interface. Then the mass slips over the belt until the friction force at the interface exceeds the tension, and the process repeats itself.

Finally, if the ground velocity ranges between $1.3510^{-7} \mathrm{~ms}^{-1}$ and $1.10^{-4} \mathrm{~ms}^{-1}$, the mechanical system is stable whatever the value of the non dimensional structural damping $\zeta$.

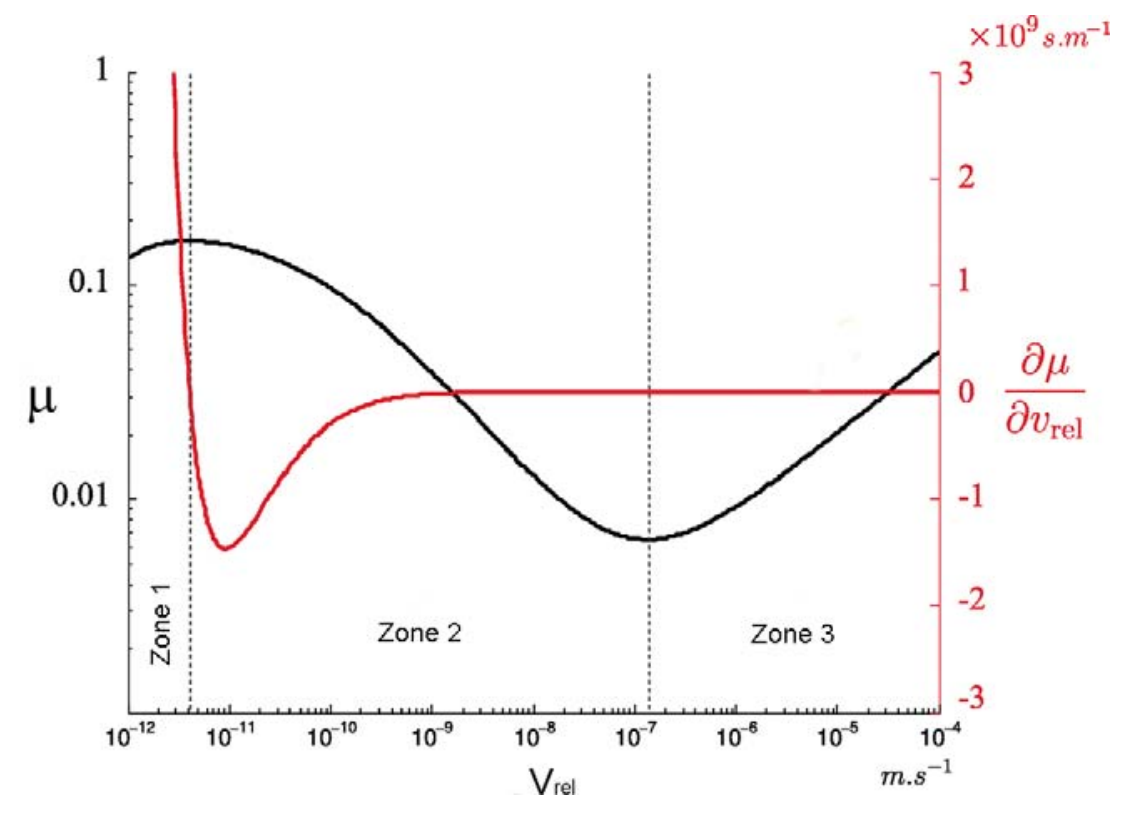

Figure 4:Evolution of the friction coefficient as a function of the sliding velocity for confined monolayers of diamine at room temperature under a normal force of $1 \mathrm{mN}$ 


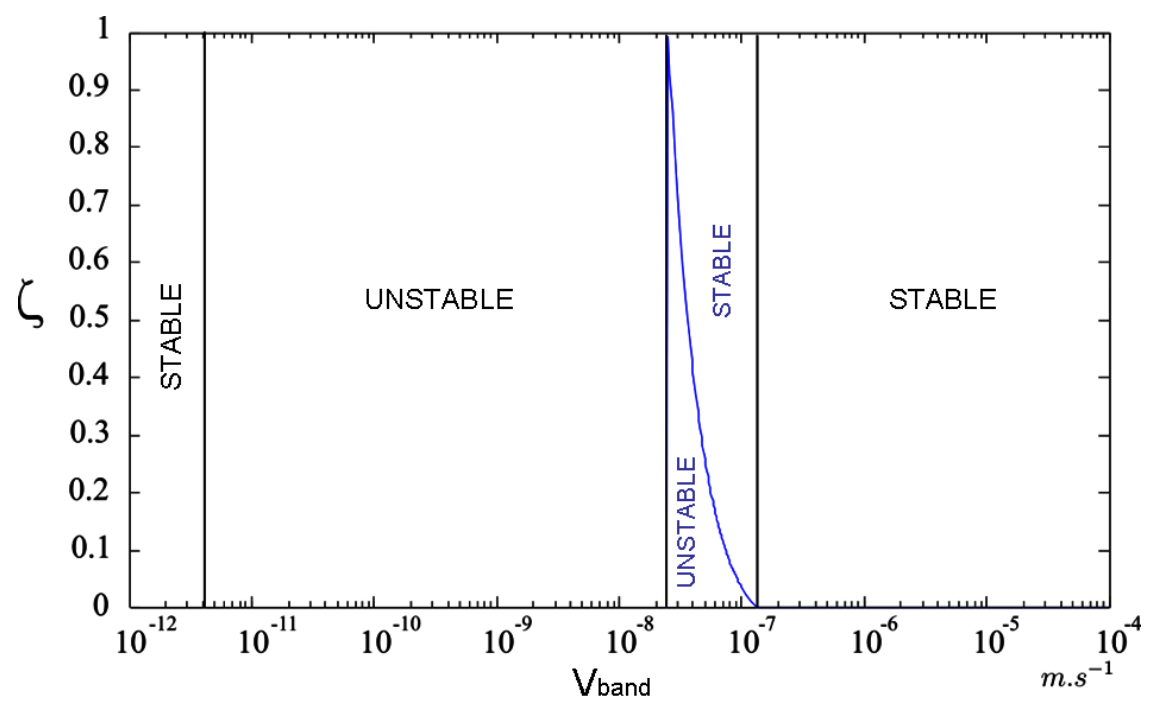

Figure 5: Stability analysis of the system
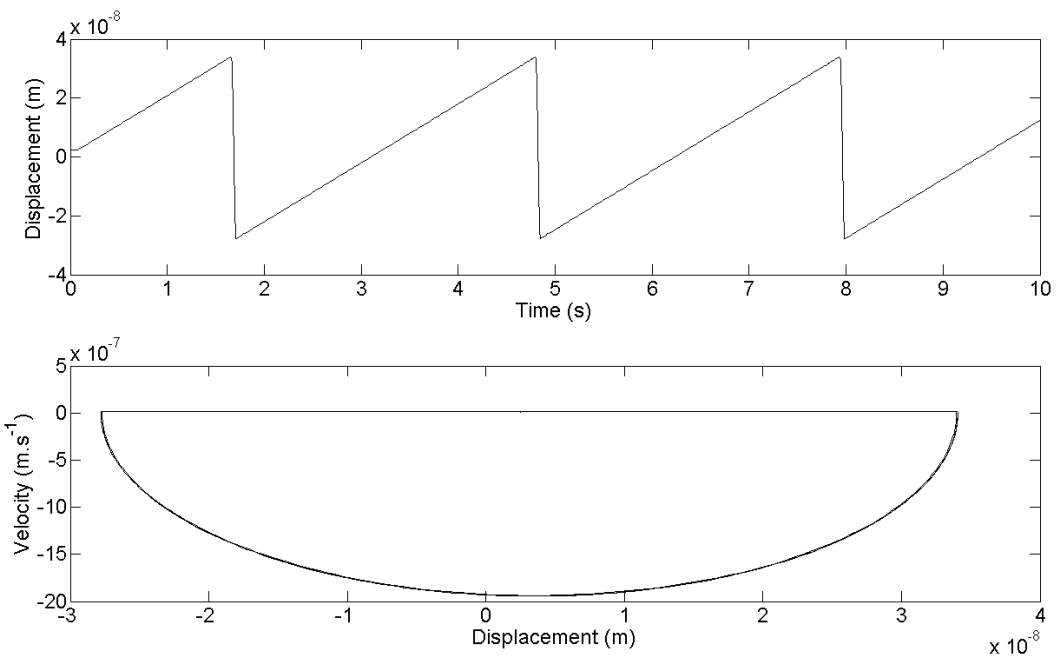

Figure 6: Stick-slip motion a) displacement vs time, b) velocity vs time

\subsection{Nonlinear behaviour of the system with a confined lubricated interface}

In real systems, sliding velocities are seldom constant throughout the operation. Since the sliding velocity seems a key factor in determining the system stability, the influence of a non-constant sliding velocity on the vibration response of the complete mechanical system with the lubricated friction model needs to be looked at in detail. In this section of the paper, the basic concept of the Continuous Wavelet Transform is first introduced before various nonlinear behaviours of the system with a confined lubricated interface are undertaken for different operating conditions.

\subsubsection{Basic theory of the wavelet analysis with Continuous Wavelet Transform}

The frequency analysis is one of the first key step in investigating of the information contained in a given signal. For steady state vibration signals, the Fast Fourier Transform (FFT) is a commonly used method: it transforms the signal from a timebased domain to a frequency-based domain. However, in the case of a non-stationary signal, the time-dependence of the frequency components is an essential point to evaluate in order to obtain a better understanding of the dynamic behaviour of the mechanical system. 
When a signal is considered as non-stationary, the characteristics of transient responses and changes in the properties of non-stationary signal of mechanical systems can be precisely analysed by means of the Continuous Wavelet Transform (CWT) [38]. The wavelet analysis transforms a signal into wavelets that are well localized both in frequency and time. The continuous Wavelet Transform (CWT) of a function $f(t)$ is defined by:

$$
W(a, b)=\int_{-\infty}^{+\infty} f(t) \psi_{a, b}^{*}(t) d t
$$

where

$$
\psi_{a, b}(t)=\frac{1}{\sqrt{a}} \psi\left(\frac{t-b}{a}\right)
$$

are the daughter wavelets (i.e. the dilated and shifted versions of the 'mother' wavelet $\psi$ that is continuous both in time and frequency). $a$ is the scale parameter, $b$ is the time parameter. The asterisk $\psi_{a, b}^{*}$ indicates the complex conjugate of $\psi_{a, b}$. The term $1 / \sqrt{a}$ ensures energy normalization across the different scales. The mother wavelet fulfils the admissibility conditions [39] i.e. $0<C_{\psi}<+\infty$ where $C_{\psi}$ defines the admissibility constant $C_{\psi}=\int_{-\infty}^{+\infty} \frac{|\hat{\psi}(\omega)|^{2}}{|\omega|} d \omega$ with $\hat{\psi}$ the Fourier transform of $\psi$. Many types of wavelets with their own features and performance in time and frequency domains have been developed. One of the most widely used mother wavelets is the Morlet wavelet defined as follows in the time domain:

$$
\psi_{0}(\alpha)=\pi^{-\frac{1}{4}} e^{i m \alpha} e^{-\frac{\alpha^{2}}{2}}
$$

where $\mathrm{m}$ is the wave-number, $\alpha$ is a non-dimensional time parameter, and $\mathrm{i}$ defines the complex number. The wavelet function contains unit energy at every scale due to the normalization of the mother wavelet. In practice, the wavelet power spectrum is used because of its analogy with the Fourier power spectrum. For this study, part of the Continuous Wavelet Transform software includes code originally written by Torrence and Compo [40].

\subsubsection{Effects of variable physical parameters}

To estimate more precisely the vibration phenomena, a series of numerical tests are performed for different operating conditions. We will consider that the belt velocity changes over regular cycles between V1 and V2 as presented in Figure 7. V1 and V2 are respectively chosen in the unstable and stable zones. The influence of the variation of the ramp time $\Delta$ Tramp, time of constant belt velocity $T c$, the minimum and maximum belt velocity $\mathrm{V} 1$ and $\mathrm{V} 2$ will be more particularly analysed. All the cases studied here are reported in Table 1 . For each case, the equivalent frequency of the belt velocity $(\mathrm{Hz})$ that is defined by $1 /$ Tbelt (with Tbelt $=\Delta$ Tramp $+\mathrm{Tc}$ ) is also given in Table 1 . The initial conditions $x_{\text {initial }}$ used in time integrations have been chosen in order to represent a small perturbation of $1 \%$ around the equilibrium point, i.e. $\mathrm{x}_{\text {initial }}=1.01 \mathrm{x}_{0}$.

First of all, Figures 8 illustrate the effect of the time of constant belt velocity Tc when other parameters are held constant (cases 1 to 3 ). Figures 8(a), (c) and (e) present the time-history response of the mechanical system with the frequency content via Continuous Wavelet Transform (CWT). The limit cycles are given in Figures 8(b), (d) and (f). For the first two cases, the dynamics of the response appears to be simple as it is composed of two domains: first, the oscillation amplitude increases before the oscillations stabilize and stationary amplitudes are reached. Moreover, an overshoot is 
observed in the transient regime for the first case, between t ranging from 0.5 to 1.5 $\mathrm{s}$ as indicated in Figure 8(a). By showing the frequencies occurring in the dynamical response, the Continuous Wavelet Transform reveals that the response is composed of two frequencies for the first case, at around $4.76 \mathrm{~Hz}$ and $9.52 \mathrm{~Hz}$ as indicated in Figure $8(a)$, while it consists of three frequencies for the second case at around $3.33 \mathrm{~Hz}$, $6.66 \mathrm{~Hz}$ and $9.99 \mathrm{~Hz}$ as indicated in Figure $8(\mathrm{~b})$. It is noted that $4.76 \mathrm{~Hz}$ and $3.33 \mathrm{~Hz}$ are the fundamental frequencies of the belt velocity for cases 1 and 2, respectively (see Table 1). Moreover, the frequency value $9.52 \mathrm{~Hz}$ corresponds to the second harmonic component of the fundamental frequency $4.76 \mathrm{~Hz}$ for case 1 . The frequency values $6.66 \mathrm{~Hz}$ and $9.99 \mathrm{~Hz}$ correspond respectively to the second and third harmonic components of the fundamental frequency $3.33 \mathrm{~Hz}$ for case 2 . So the dynamic response of the system is not only composed of the equivalent fundamental frequency of the belt velocity but also its harmonics. Even if slight differences in the frequency content and in the initial transient response are noticed, these two first cases appear to be very similar with identical amplitude levels. For case 3, the time-plot displays fluctuations in oscillations as indicated in Figure 8(e): the response of the mechanical system appears to become more complex with a succession of increase and decrease in amplitudes. These successive increase and decrease of oscillations are due to the fact that the time of constant belt velocity is sufficient enough to allow the establishment of the stability mechanism around V1 and V2. As previously explained in Section 3.1, for $\mathrm{V} 1=1.10^{-7} \mathrm{~m} \cdot \mathrm{s}^{-1}$ the system is unstable causing an increase in the response. For $\mathrm{V} 2=2.10^{-7} \mathrm{~m} \cdot \mathrm{s}^{-1}$, the system is stable leading to the decrease of the response. Finally, we detect multiple frequencies in the range $1-10 \mathrm{~Hz}$ with a predominant frequency at $10 \mathrm{~Hz}$. It should be noted that the fundamental frequency of the belt is $0.83 \mathrm{~Hz}$ for this case. Therefore the multiple frequencies observed via the wavelet spectrum correspond to the harmonic components of the frequency of the belt. The predominant frequency at $10 \mathrm{~Hz}$ is related to the $12^{\text {th }}$ harmonic component. Finally, it can be assumed that the limitation of vibration amplitudes is due to the combination of the growth rate and the evolution of the belt velocity changes that induces the successive increase and decrease of oscillations

\begin{tabular}{|c|c|c|c|c|c|}
\hline Case & $\mathrm{V} 1\left(\mathrm{~m}^{-\mathrm{s}^{-1}}\right)$ & $\mathrm{V} 2\left(\mathrm{~m} \cdot \mathrm{s}^{-1}\right)$ & $\mathrm{Tc}(\mathrm{s})$ & $\Delta$ Tramp (s) & $\begin{array}{c}\text { Frequency of the belt } \\
\text { velocity (Hz) } 1 / \text { Tbelt }\end{array}$ \\
\hline 1 & $1.10^{-7}$ & $2.10^{-7}$ & 0.01 & 0.2 & 4.76 \\
\hline 2 & $1.10^{-7}$ & $2.10^{-7}$ & 0.1 & 0.2 & 3.33 \\
\hline 3 & $1.10^{-7}$ & $2.10^{-7}$ & 1 & 0.2 & 0.83 \\
\hline 4 & $1.10^{-7}$ & $10.10^{-7}$ & 0.01 & 0.2 & 4.76 \\
\hline 5 & $1.10^{-7}$ & $10.10^{-7}$ & 0.1 & 0.2 & 3.33 \\
\hline 6 & $1.10^{-7}$ & $10.10^{-7}$ & 1 & 0.2 & 0.83 \\
\hline 7 & $1.10^{-7}$ & $10.10^{-7}$ & 0.08 & 0.02 & 20 \\
\hline 8 & $1.10^{-7}$ & $10.10^{-7}$ & 0.04 & 0.01 & 33.3 \\
\hline 9 & $1.10^{-7}$ & $2.10^{-7}$ & 0.01 & 0.02 & 33.3 \\
\hline 10 & $1.10^{-7}$ & $10.10^{-7}$ & 0.01 & 0.02 & \\
\hline
\end{tabular}

Table 1: Operating conditions for the belt velocity changes over regular cycles 


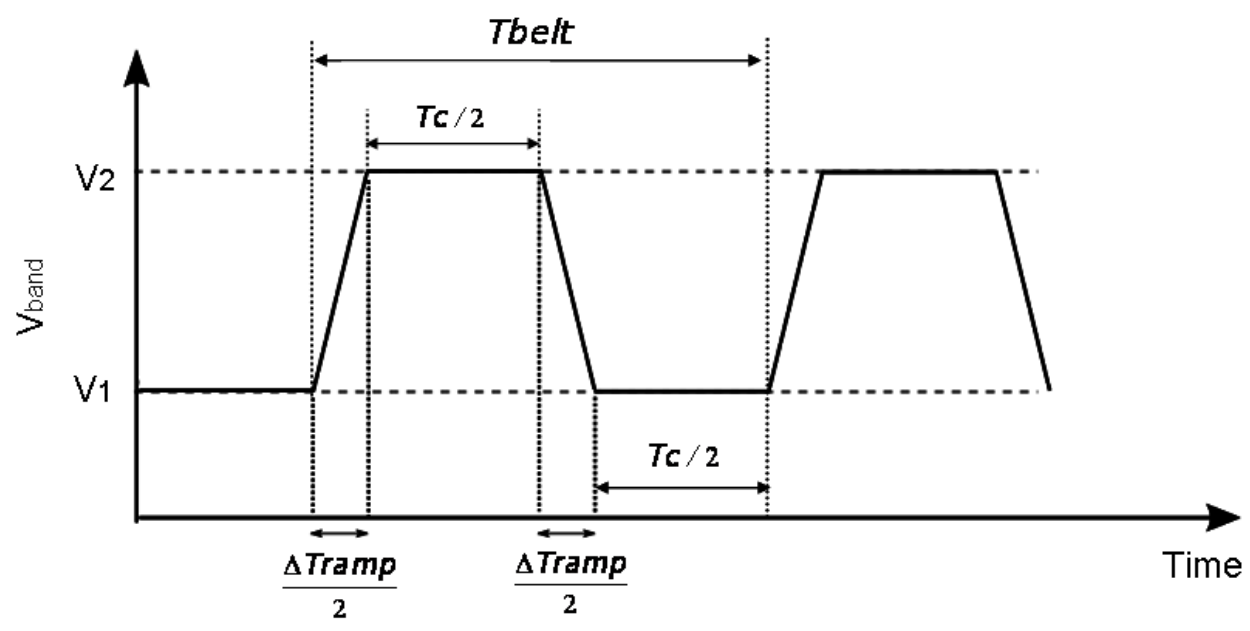

Figure 7: Belt velocity changes over regular cycles. Tbelt corresponds to the periodicity of a cycle, $\Delta$ Tramp is the ramp time and Tc is the time of the plateau of constant velocity. V1 and V2 are respectively the minimum and the maximum belt velocity reached in a cycle.

Then, we propose to analyse the effects of the maximum belt velocity V2. To do so, the previous cases are reconducted by changing only this parameter as indicated in Table 1. Figures 9(a) and (b) illustrate the time history, frequency spectrum via CWT and the limit cycles for case 4. Apart from the initial transient behaviour and the vibration amplitudes, the simple vibration behaviour of the system and the frequency content can be compared with that of case 1 . The predominant frequency is identified at around $4.76 \mathrm{~Hz}$ in this case, with two small contributions around $9.52 \mathrm{~Hz}$ and $14.28 \mathrm{~Hz}$. As previously explained, we observed the equivalent fundamental frequency of the belt velocity and its second and third harmonic components. For case 5, the vibration develops with a classically increase, quite similar to that observed for case 2. However, it is noteworthy that the quasi-periodic amplitudes and velocities are larger. The system response is composed of two predominant frequencies at around $3.33 \mathrm{~Hz}$ and $9.99 \mathrm{~Hz}$ corresponding to the fundamental frequency of the belt velocity and its third harmonic component.

For case 6, the time plots of Figure 9(e) and (f) show significantly different behaviours compared to the previous cases. As seen in Figure 9(f), the complete dynamics appears to comprise two specific limit cycles with transitions from one to the other: for the first one (i.e. the left limit cycle in Figure 9(f)), a vibration develops and increases, while oscillations decrease for the second limit cycles. This behaviour can be compared with the nonlinear behaviour previously observed for case 3 : the time of constant belt velocity is sufficient to allow the establishment of the mechanism around V1 and V2. The novelty compared to the previous case 3 is that these two oscillations and limit cycles are clearly separated in phase plot with jumps of amplitude responses of the system. Moreover, it can be observed that the amplitude level is more important. As previously explained, the limitation of the vibration amplitudes is due to the growth rate and the evolution of the belt velocity changes. Finally, the detailed CWT of Figure $9(\mathrm{e})$ gives a view of all the frequencies between $0.8 \mathrm{~Hz}$ and $10 \mathrm{~Hz}$ making up the signal: the frequency content is extremely rich with four predominant contributions at $0.83 \mathrm{~Hz}, 2.49 \mathrm{~Hz}, 4.15 \mathrm{~Hz}$ and $9.96 \mathrm{~Hz}$ i.e. the fundamental frequency of the belt velocity and its $3^{\text {rd }}, 5^{\text {th }}$ and $12^{\text {th }}$ harmonic components, respectively.

From these examples, it can be concluded that variations of the time of constant belt velocity Tc, the minimum and maximum belt velocities $\mathrm{V} 1$ and $\mathrm{V} 2$ can drastically affect not only the vibration amplitudes of the mechanical system but also the 
frequency content and the transient behaviour with more or less complex nonlinear behaviours. The frequency content can be composed of multiple frequencies that correspond to the equivalent fundamental frequency of the belt velocity and its harmonic components.
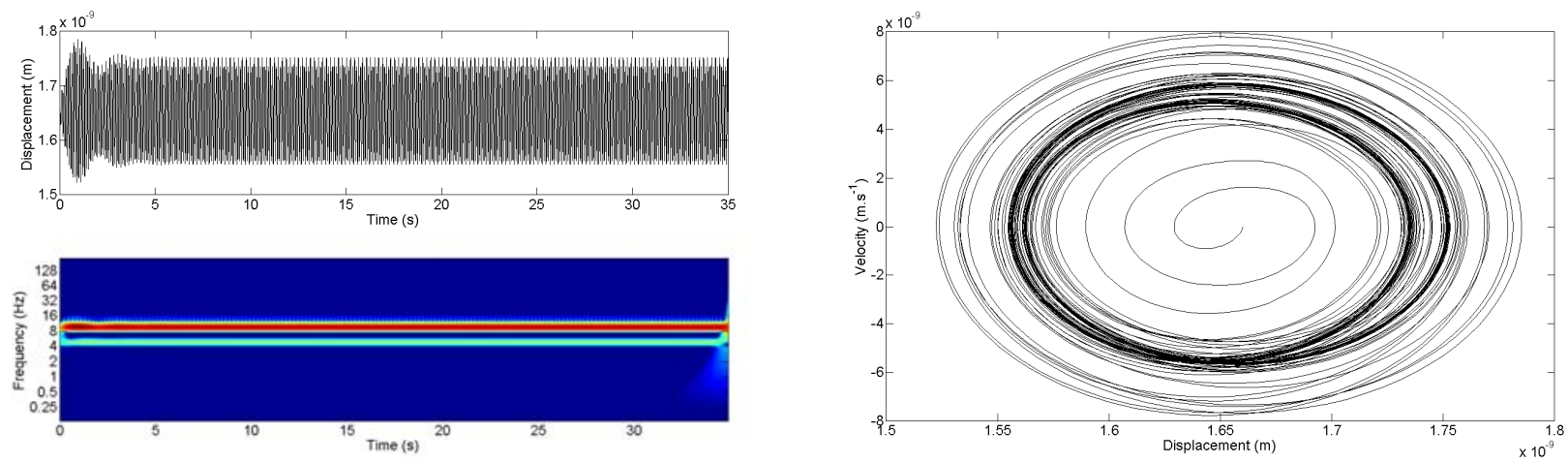

(a)

(b)
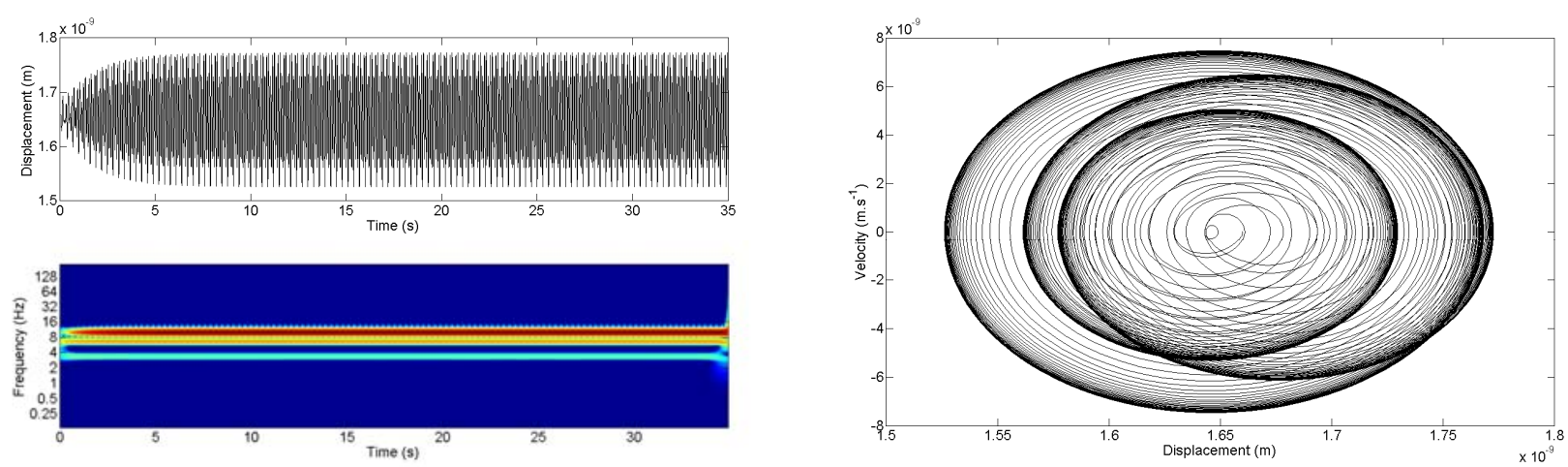

(c)

(d)
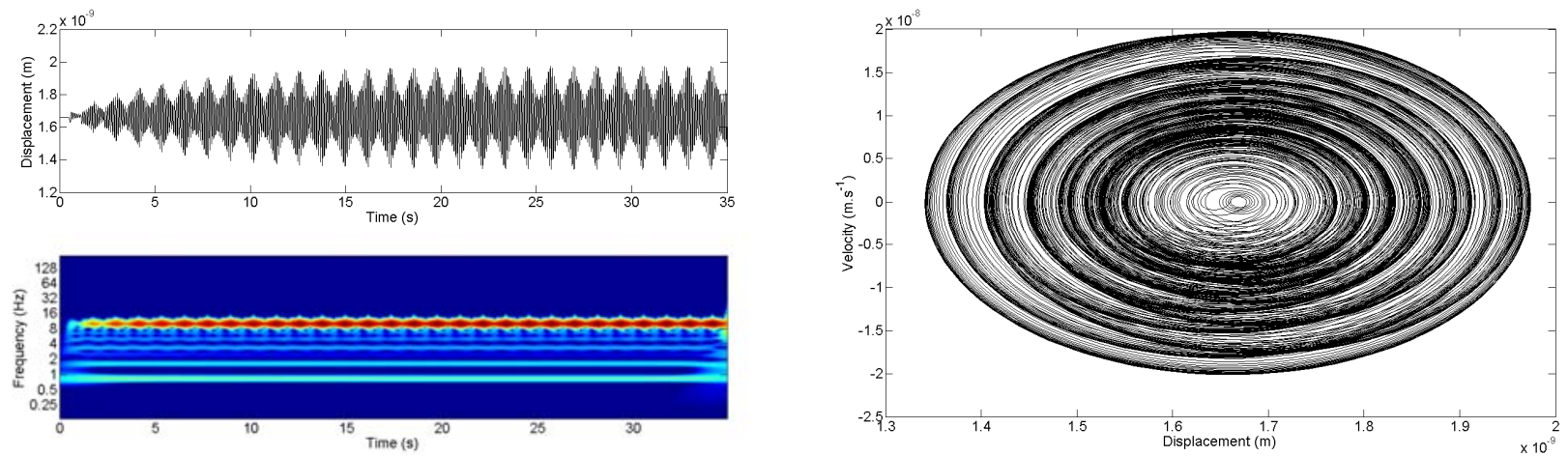

(e)

Figure 8: Nonlinear dynamics of the system for cases 1, 2 and 3 (a) time history and wavelet power spectrum for case 1 ; (b) phase plot for case 1 ; (c) time history and wavelet power spectrum for case 2; (d) phase plot for case 2; (e) time history and wavelet power spectrum for case 3; (f) phase plot for case 3 

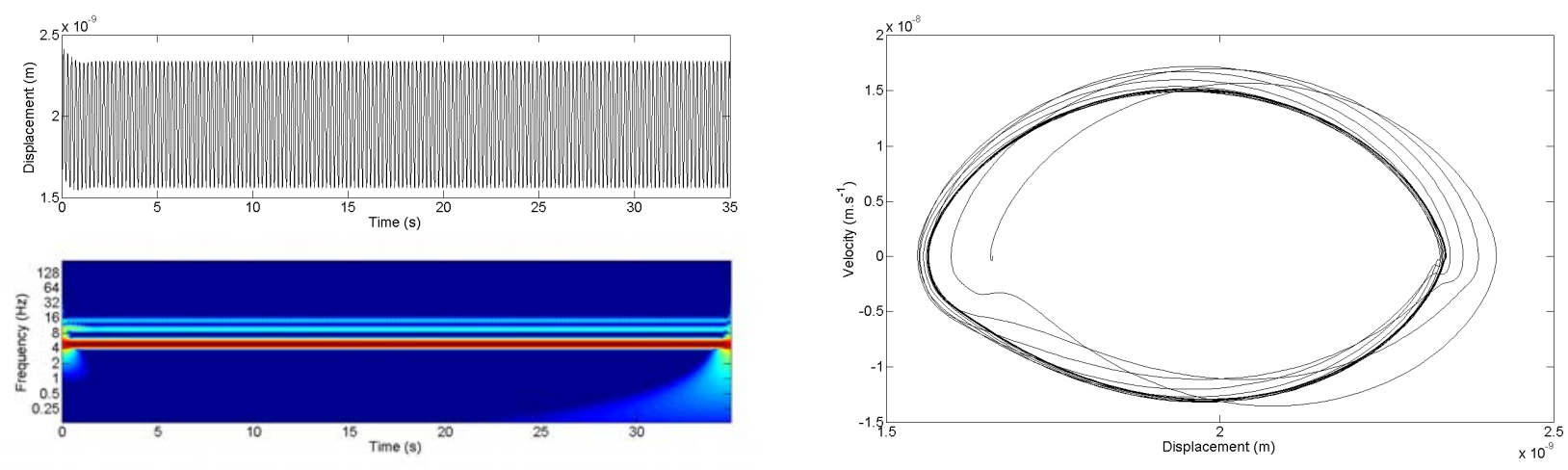

(a)

(b)
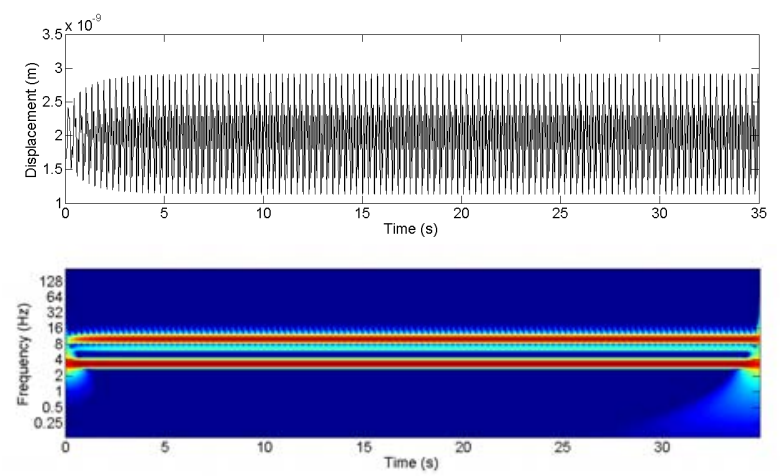

(c)

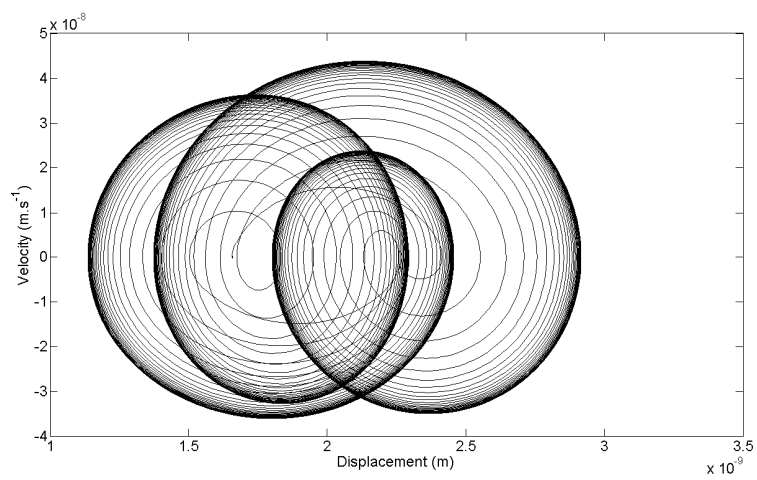

(d)
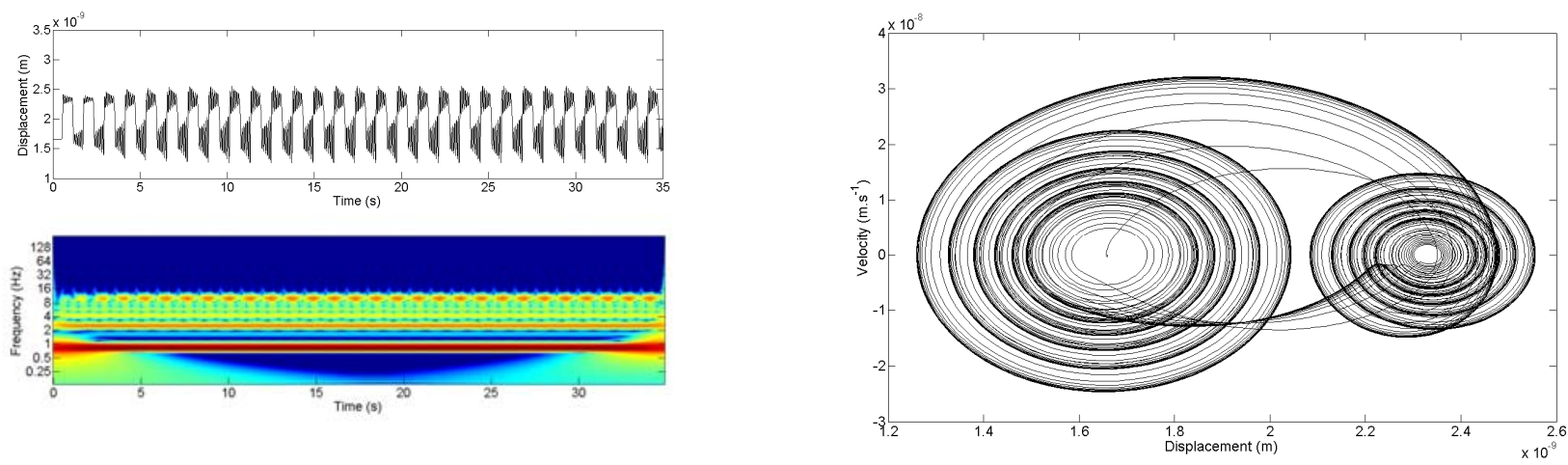

(e)

(f)

Figure 9: Nonlinear dynamics of the system for cases 4, 5 and 6 (a) time history and wavelet power spectrum for case 4 ; (b) phase plot for case 4 ; (c) time history and wavelet power spectrum for case 5; (d) phase plot for case 5; (e) time history and wavelet power spectrum for case 6; (f) phase plot for case 6

Considering the previous results, a specific case is now presented and discussed: the belt velocity changes are always performed over regular cycles, but the frequency of these regular cycles corresponds to the fundamental frequency of the mechanical system. Figures 10 (a) and (b) show the time plot, the associated wavelet power 
spectrum and the phase plot for this case 7. It can be seen that the displacement increases until periodic oscillations are obtained. As indicated by the wavelet power spectrum, the response of the system is mono-harmonic at $10 \mathrm{~Hz}$. This phenomenon can be interpreted as a classical dynamical behaviour of a mechanical system undergoing external excitation synchronized on its own frequency resonance. In comparison with case 5 (i.e. the same operating conditions expect for the ramp time $\Delta$ Tramp), it is clearly shown that the oscillation magnitudes are eight times higher here. This example perfectly illustrates the need to avoid a match between the excitation frequency and the resonant frequency of the mechanical system.

In conclusion, it can be noted that for each case the vibration amplitude depends on the growth rate of the oscillation, the evolution of the belt velocity changes over regular cycles (i.e. the difference between V1 and V2) and the frequency of these regular cycles of the belt (due to the synchronisation or not with the fundamental frequency of the mechanical system).

\subsubsection{How does the local dynamics control the macroscopic behaviour?}

By varying $\Delta$ Tramp and Tc, other behaviours such as stick-slip phenomenon, may also be triggered. For example, Figures $11(\mathrm{a})$ and (b) show the transient and stationary quasi-periodic vibrations corresponding to case 8 . The operating parameters are similar to those of cases 4-7 except for the value of the ramp time $\Delta$ Tramp and the time of constant belt velocity Tc. Firstly, a decrease of the transient oscillations for $t$ inferior to $2 \mathrm{~s}$ is followed by a very fast increase for $t$ higher than $4 \mathrm{~s}$, as illustrated in Figure $11(\mathrm{a})$. Two predominant frequencies at $10 \mathrm{~Hz}$ and $20 \mathrm{~Hz}$ are present during this first part of the system behaviour. Then, during the second part of the transient oscillations (for $t>4 \mathrm{~s}$ ), stationary oscillations are seen without modification in the frequency content. Figure 11 (b) shows that this second part of the system behaviour is governed by a stick-slip motion when the velocity remains constant for a varying displacement. As previously explained in Section 3.1, the following repetitive process occurs: when the tension force reaches the value of the friction force at the lubricated interface the mass of the mechanical system slips over the belt. When the friction force at the interface exceeds the tension, the mass sticks.

For this case, it appears that the two frequencies correspond to the fundamental frequency of the mechanical system and the equivalent frequency of the belt velocity.

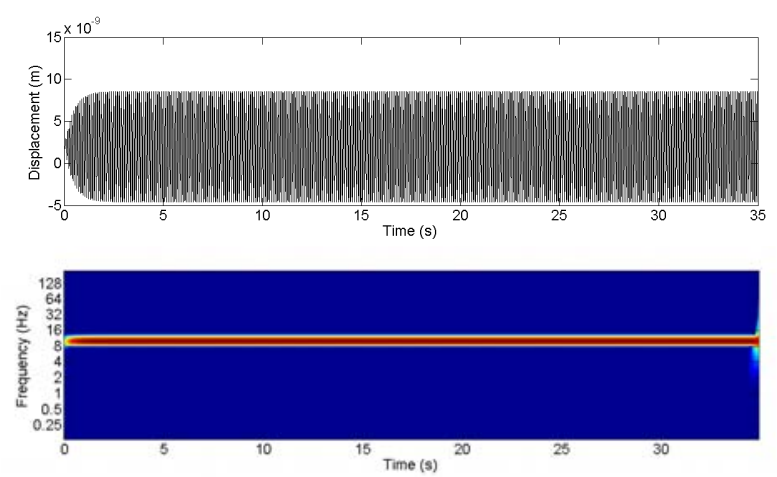

(a)

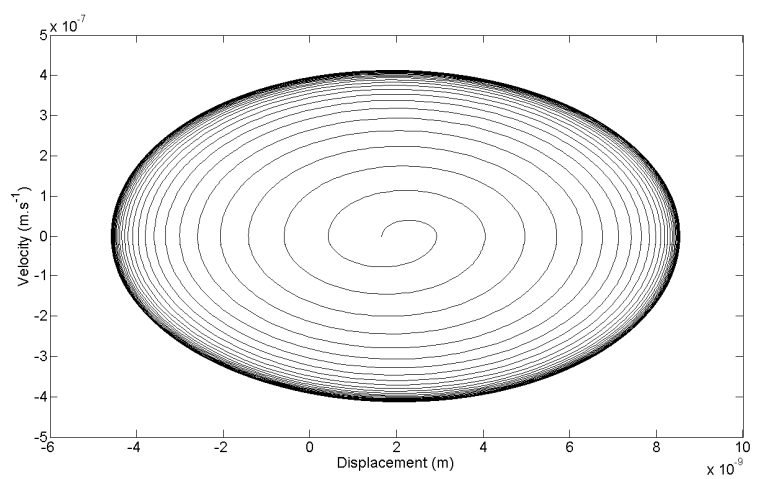

(b)

Figure 10: Nonlinear dynamics of the system for case 7 (a) time history and wavelet power spectrum; (b) phase plot 


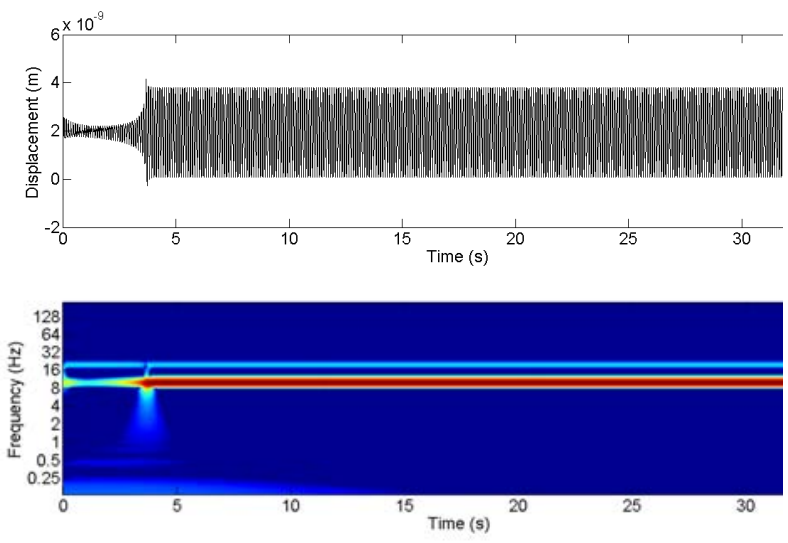

(a)

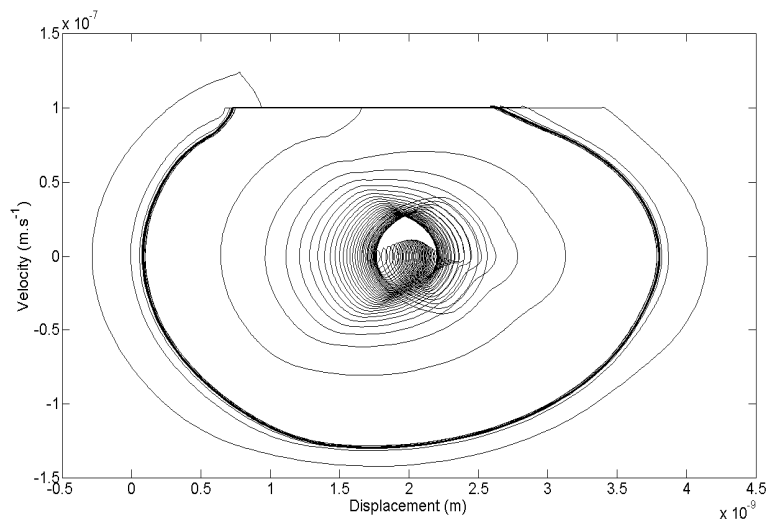

(b)

Figure 11: Nonlinear dynamics of the system for case 8 (a) time history and wavelet power spectrum; (b) phase plot
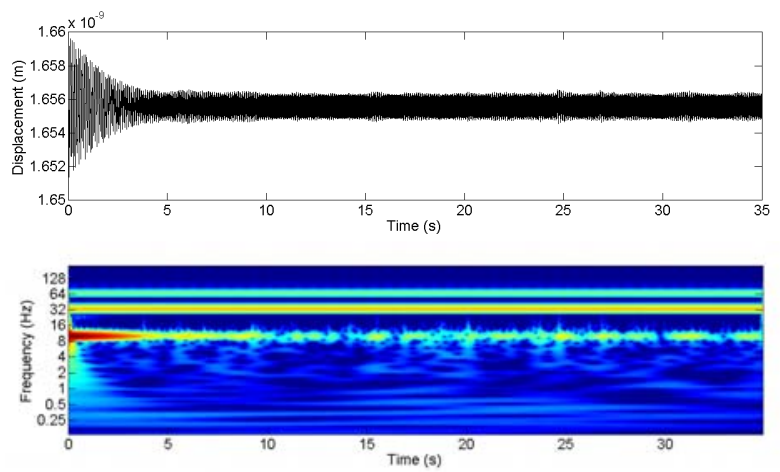

(a)
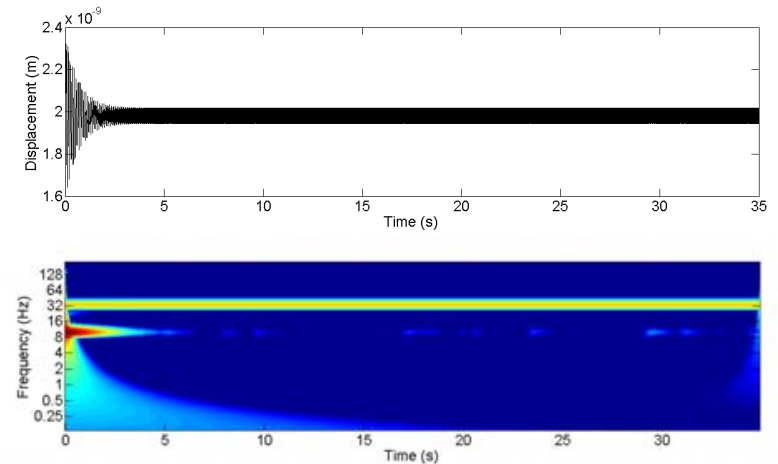

(c)

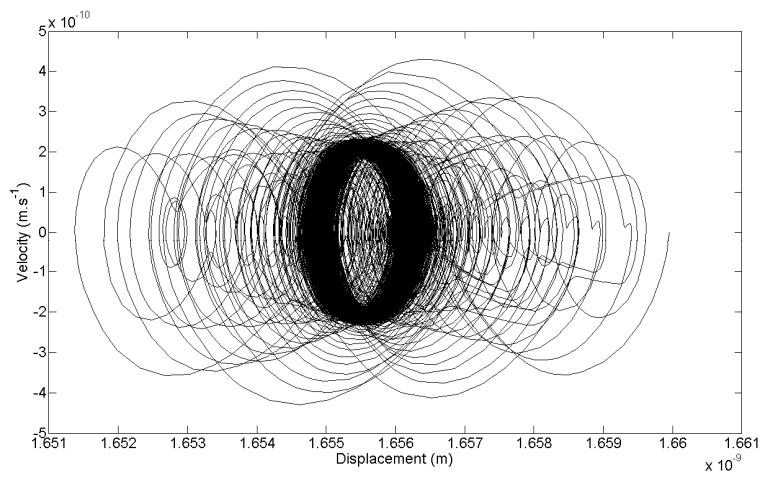

(b)

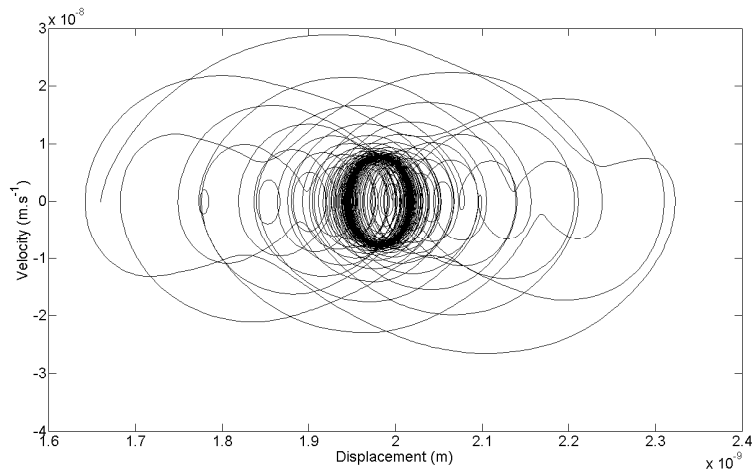

(d)

Figure 12: Nonlinear dynamics of the system for cases 9 and 10 (a) time history and wavelet power spectrum for case 9; (b) phase plot for case 9; (c) time history and wavelet power spectrum for case $10 ;(d)$ phase plot for case 10 
Another interesting phenomenon can be put forward. We show that one can attenuate the macroscopic dynamic behaviour by controlling the local lubricated friction dynamics: under certain operating conditions, the movement of the belt and the associated non-constant sliding velocity may lead to a decrease and an attenuation of stationary vibrations of the mechanical system with the lubricated interface. This fact is illustrated in Figures 12 with the operating conditions given in Table 1 corresponding to cases 9 and 10. For the reader's understanding, cases 9 and 10 can be compared to the cases 2 and 5, respectively: all the operating parameters are similar to these previous cases except for the value of the ramp time $\Delta$ Tramp. In comparison with the previous cases 2 and 5, two behaviours can be identified when examining the time history of the system response. First, a very fast decrease of the transient oscillations is observed; then, small residual stationary oscillations are depicted. Showing the CWT analysis, the resonance frequency of the mechanical system at $10 \mathrm{~Hz}$, which is present in the first part of the transient vibrations (for $t$ lower than $10 \mathrm{~s}$ for case 9 and for $t$ inferior to $5 \mathrm{~s}$ for case 10), disappears in the second part of the time plot and stationary oscillations occur for $t>10 \mathrm{~s}$ for case 9 and for $t>5 \mathrm{~s}$ for case 10 .

As indicated by the CWT analysis (see Figures 12(a) and (c)), the frequency content is composed of only the fundamental frequency at $33 \mathrm{~Hz}$ for case 9 , and only the fundamental frequency at $33 \mathrm{~Hz}$ and its second harmonic component at $66 \mathrm{~Hz}$ for case 10. So, the previous observations can be explained by the fact that the system is first governed by its fundamental frequency and then the system is excited by the frequency of the moving belt. Due to the fact that the fundamental frequency of the system and the frequency of the belt are not in concordance, small vibration amplitudes are observed. Moreover, the mechanical system cannot be excited by the harmonic components of the belt velocity that are higher than the fundamental frequency of the system. This is one way to reduce or avoid amplitudes of the mechanical system with a lubricated interface.

\subsubsection{Evolution of the friction coefficient}

It can be interesting to investigate the evolution of the friction coefficient versus time for each previous case. Such information can be useful to answer the following questions:

- Does the friction coefficient fluctuate more or less during the transient or stationary responses of the mechanical system?

- Is it possible to clearly understand the variation of the friction coefficient during the system response?

To answer these questions, Figures 13 and 14 give the evolution of the friction coefficient versus time for cases $1-6$ and cases 7-10, respectively. Moreover, the velocity of the mechanical system $\dot{x}(t)$ and the band velocity $v_{\text {band }}(t)$ (i.e. velocity changes over regular cycles between V1 and V2) are also given to better analyse the fluctuation of the friction coefficient with time.

Considering all cases in Figures 13 and 14, various situations are found. First of all, it is observed that the differences between the minimum and maximum values of the friction coefficient during time can be very small between $\left[6.510^{-3} ; 6.610^{-3}\right.$ ] (see for example cases 1, 2, 3, and 9 in Figures 13(a), 13(b), 13(c) and 14(c), respectively). In some other cases, this fluctuation is more important between $\left[6.10^{-3} ; 10.10^{-3}\right]$ (see for example cases 4-6, 7 and 10 in Figures 13(d-f) ,14(a) and 14(d), respectively). These observations can be explained by the fact that the velocity fluctuation of the belt $v_{\text {band }}(t)$ is more important than the velocity evolution of the mechanical system 
$\dot{x}(t)$ (as illustrated in Figures 13 and 14 and $14(c-d)$ ). So the evolution of the friction coefficient seems mainly governed by the evolution of the belt velocity $v_{\text {band }}(t)$ (via equation 4). For case 8 where stick-slip occurs (Figure 14(b)), fluctuation of the friction coefficient is very high between $\left[6.510^{-3} ; 0.16\right]$ due to the high variation of the velocity of the mechanical system $\dot{x}(t)$ from $-1.10^{-7} \mathrm{~ms}^{-1}$ to $1.10^{-7} \mathrm{~ms}^{-1}$. Consequently, the values of the velocity $\dot{x}(t)$ and the belt velocity $v_{\text {band }}(t)$ can be of the same order of magnitude: this induces a relative velocity of the mass $v_{\text {rel }}=v_{\text {band }}-\dot{x}$ equal to zero resulting in a high value of the friction coefficient, as indicated in Figure 4 . It can be concluded that the friction coefficient fluctuates more or less during the transient or stationary responses of the mechanical system due to the fluctuation of both the system velocity $\dot{x}(t)$ and the belt velocity $v_{\text {band }}(t)$.

In addition, the evolution of the friction coefficient can also be more or less complex. These different behaviours depend on the trends of the two velocities (i.e. system velocity $\dot{x}(t)$ and belt velocity $v_{\text {band }}(t)$ ). For example, if the temporal evolution of the belt velocity $\left.v_{\text {band }}(t)\right)$ is really much more important than the evolution of the system velocity $\dot{x}(t)$, the vibration behaviour of the friction coefficient follows the evolution of the belt velocity $v_{\text {band }}(t)$ (see for example cases $4,5,6,7,9$ and 10 in Figures 13(d), 13(e), 13(f), 14(a), 14(c) and 14(d)). In other cases, the temporal evolution of the friction coefficient is more complex, as illustrated in Figures 13(a), 13(b) and 13(c) (for cases 1, 2 and 3). For cases 1 and 2, it is shown that the friction coefficient fluctuates due to the periodic evolution of both the belt velocity $v_{\text {band }}(t)$ ) and the system velocity $\dot{x}(t)$. For case 3 , the temporal fluctuations of the friction coefficient are even more pronounced and complex: the global evolution of the friction coefficient follows the temporal evolution of the belt velocity $v_{\text {band }}(t)$; additionally, a fluctuation of the friction coefficient around this 'global evolution' is observed. This supplementary evolution is governed by that of the system velocity $\dot{x}(t)$ : an increase in amplitude of the system velocity $\dot{x}(t)$ results in fluctuations of the friction coefficient around the temporal 'global value' (see for example evolution of the signal for $t=[3.6 ; 4.1] \mathrm{s}$ or $\mathrm{t}=[6 ; 6.5] \mathrm{s})$. In contrast, fluctuations of the friction coefficient decrease around the 'global value' when amplitudes of the system velocity $\dot{x}(t)$ decrease (see for example evolution of the signal for $t=[5.4 ; 5.9] \mathrm{s}$ or $t=[7.8 ; 8.3] \mathrm{s}$ ).

In conclusion, variations and temporal changes of the friction coefficient are consistent with the temporal evolutions of both the belt velocity $\left.v_{\text {band }}(t)\right)$ and the system velocity $\dot{x}(t)$. It is recalled that the others parameters of Equation 4 (for the friction law) are assumed to be constant for the present study. It is also seen that evolution of the friction coefficient can be more or less complex during time due to the specific vibrational behaviour of the mechanical system. 

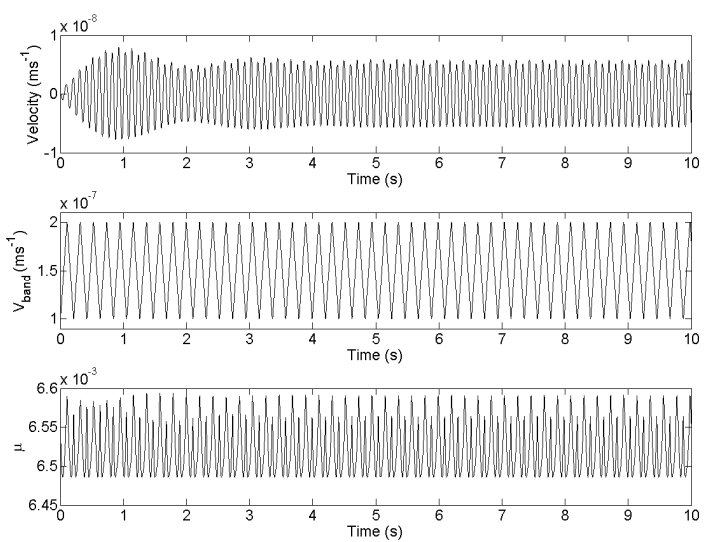

(a)
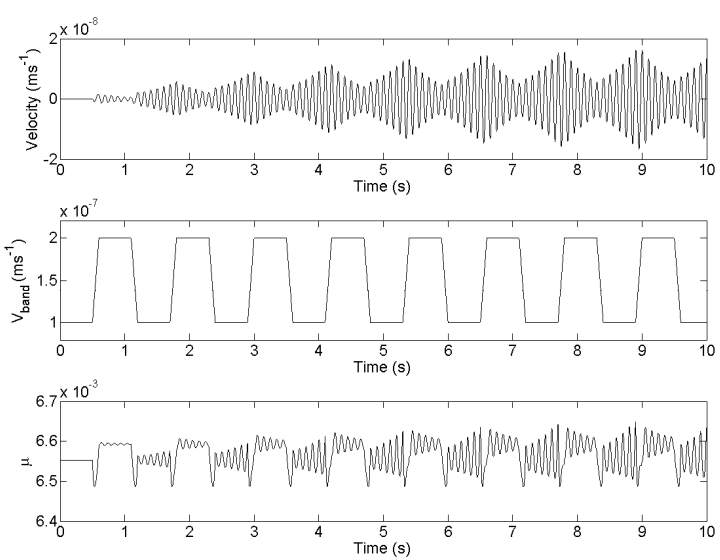

(c)

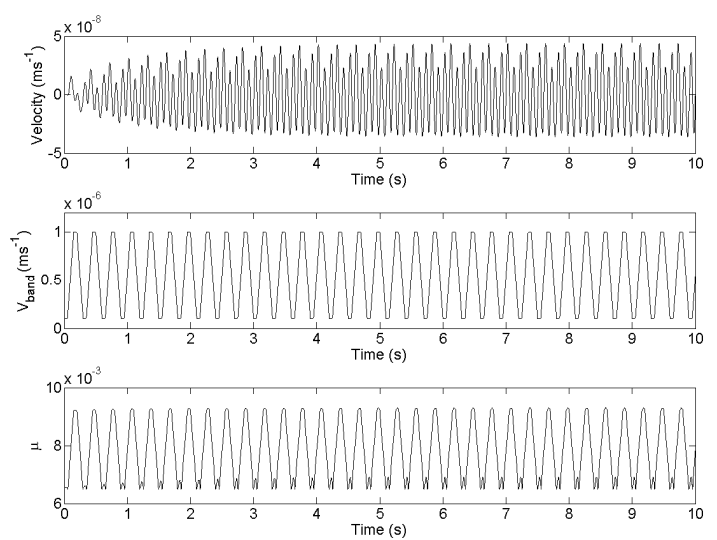

(e)
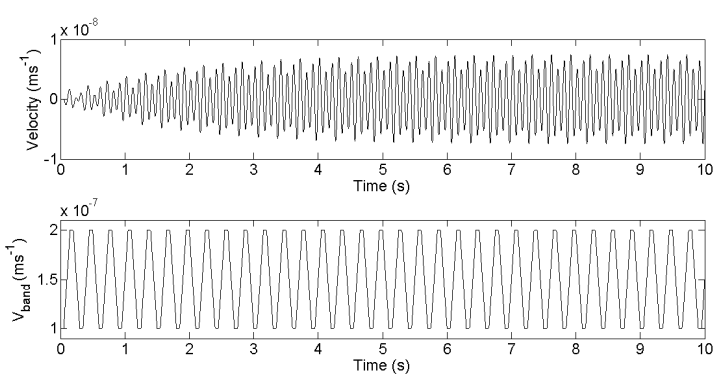

$6.7 \times 10^{-3}$

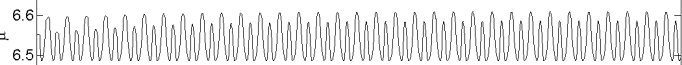

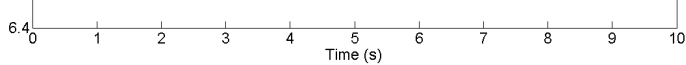

(b)

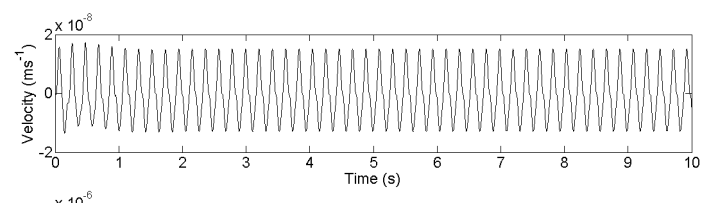

$\times 10^{-6}$
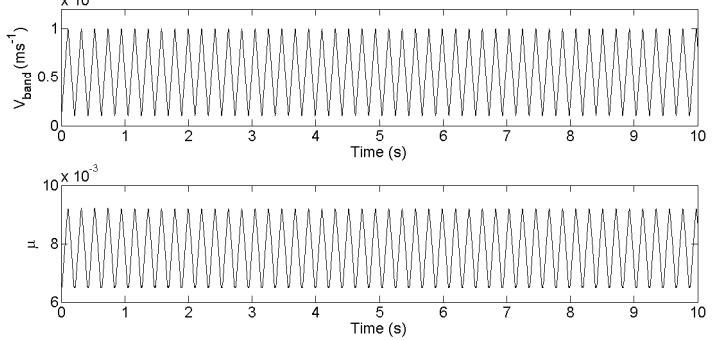

(d)
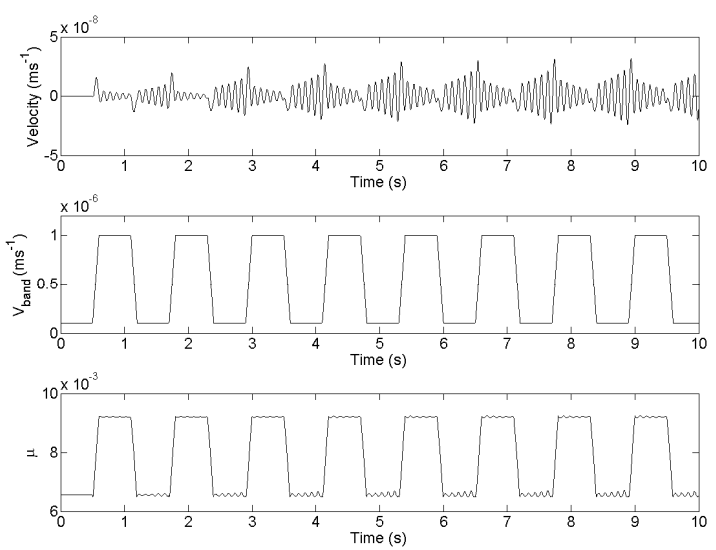

(f)

Figure 13: Temporal evolution of the system velocity, the belt velocity and the friction coefficient (a) case 1 ; (b) case 2; (c) case 3; (d) case 4; (e) case 5; (f) case 6 

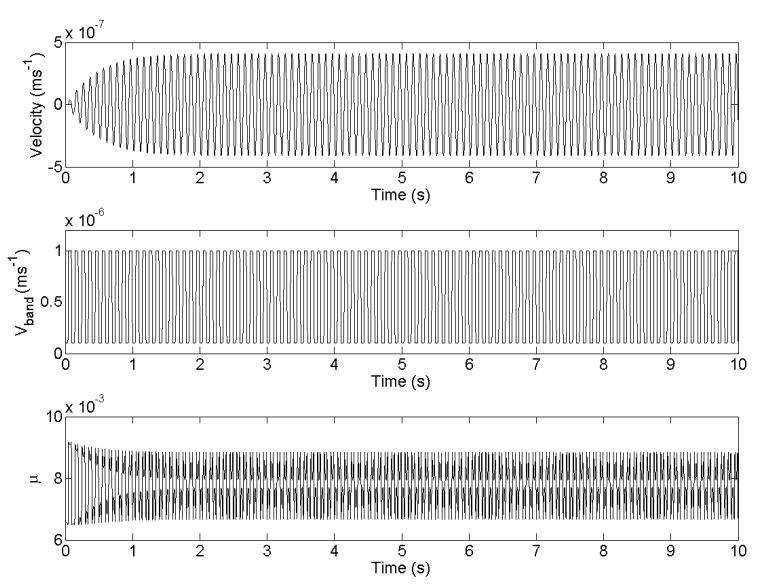

(a)
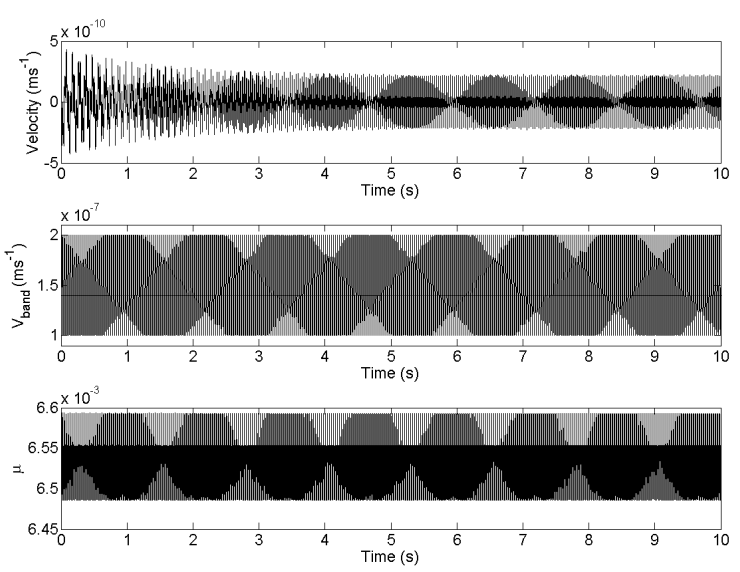

(c)
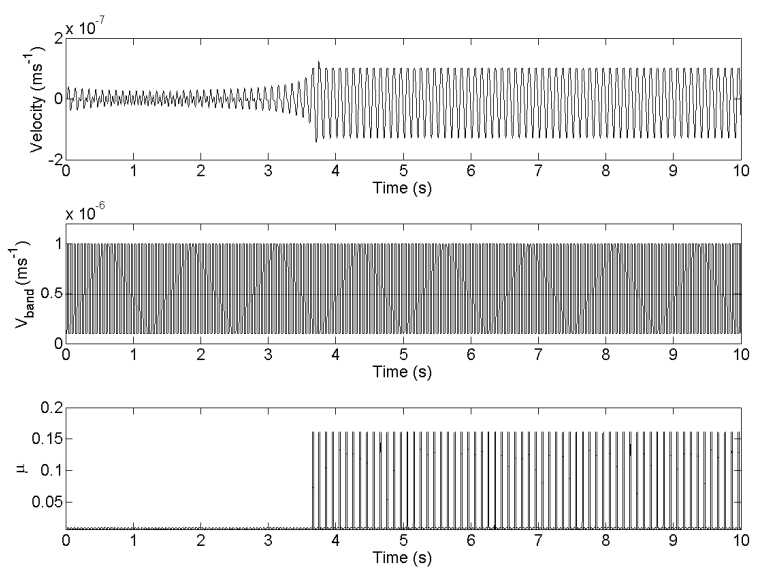

(b)
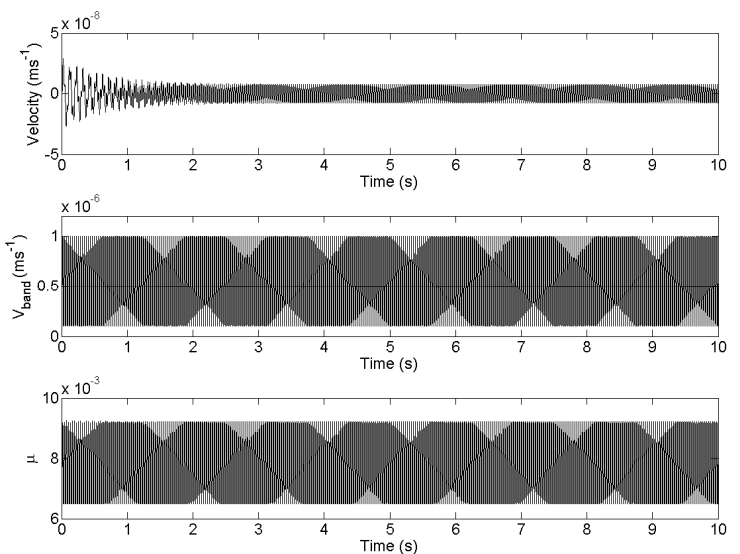

(d)

Figure 14: Temporal evolution of the system velocity, the belt velocity and the friction coefficient (a) case 7; (b) case 8; (c) case 9; (d) case 10

\section{Conclusions}

This study takes place in the context of the local dynamic responses of a mechanical system with lubricated interface. A rather simple mechanical model, that is to say a single-degree of freedom block on a moving belt, was deliberately selected. Nevertheless the originality of this work lies on the choice of a realistic friction law, issued from experimental results, that accounts for the physical dissipation mechanisms within the lubricated interface. This coupled approach allows us to develop a complete numerical investigation in order to bring a better insight into the dynamic behaviour of such a system. Not only the stability analysis of the system, but also the vibrational oscillations and a complete analysis of the frequency content via the Continuous Wavelet Transform were presented and discussed through various operating conditions. More particularly, the influence of a non-constant velocity of the belt is undertaken.

We clearly demonstrated the impact of the periodicity of velocity cycles, as well as the values of minimum and maximum velocities reached by the belt, on the complexity of the nonlinear behaviour in terms of vibration amplitudes and frequency content. We also illustrated the consequence of a match between the excitation frequency and the resonant frequency of the mechanical system. Last but not least, we showed that the 
local dynamics can control the macroscopic behaviour by triggering stick-slip phenomenon or attenuating the vibrations.

Giving an exhaustive list of topics of interest for future developments is not possible. However, some non-exhaustive interesting further studies can be considered:

- the improvement of correlations between experimental and numerical analysis for a better understanding of the nonlinear behaviour of lubricated mechanical systems;

- the extension of the proposed numerical process for complex industrial systems.

On a more fundamental aspect, one might wonder how to take advantage of this correlation between local dynamics and macroscopic nonlinear behaviour. Is it possible to control the evolution of the local dynamics with an adequate choice of monolayers in terms of viscoelastic properties and adsorption characteristics, and consequently the vibrational behaviour of a lubricated mechanical system?

\section{$\underline{\text { References }}$}

[1] Sinou JJ, Thouverez F, Jezequel L. Methods to reduce nonlinear mechanical systems for instability computation. Archives of computational methods in engineering 2004; 11: 257-344.

[2] Sinou J]. Transient nonlinear dynamic analysis of automotive disc brake squeal On the need to consider both stability and nonlinear analysis. Mechanics Research Communications 2010; 37: 96-105.

[3] Brizard D, Chiello O, Sinou J], Lorang X. Performances of some reduced bases for the stability analysis of a disc/pads system in sliding contact. Journal of Sound and Vibration 2011; 330: 703-720.

[4] Loyer A, Sinou JJ, Chiello O, Lorang X. Study of nonlinear behaviours and modal reductions for friction destabilized systems. Application to an elastic layer. Journal of Sound and Vibration 2012; 331: 1011-1041.

[5] Spurr RT. A theory of brake squeal. Proc. IMechE 1961-1962; 1 :33-40.

[6] Jarvis RP, Mills B. Vibration Induced by Dry Friction. Procs. of the Institution of Mechanical Engineers 1964; 178: 847-866.

[7] Israelachvili JN, Mc Guiggan P, Homola AM. Dynamic Properties of Molecularly Thin Liquid Films. Science 1988; 240: 189-191.

[8] Georges JM, Tonck A, Mazuyer D. Interfacial friction of wetted monolayers. Wear 1994; 175: 59-62.

[9] Carpick RW, Agraït N, Ogletree DF, Salmeron M. Measurement of interfacial shear (friction) with an ultrahigh vacuum atomic force microscope. J. Vac. Sci. Technol. 1996; B14: 1289-1295.

[10] Granick S. Motions and relaxations of confined liquids. Science 1991; 253: 13741379.

[11] Gee ML, Mc Guiggan P, Israelachvili JN, Homola AM. Liquid to solidlike transitions of molecularly thin films under shear. J. Chem. Phys. 1990; 93: 1895-1906.

[12] Thompson PA, Robbins MO. Shear flow near solids: Epitaxial order and flow boundary conditions. Phys. Rev. 1990; A41: 6830-6837.

[13] Schoen M, Rhykerd CL, Diesler DJ, Cushman JH. Shear forces in molecularly thin films. Science 1989; 254: 1223-1225.

[14] Thompson PA, Grest GS, Robbins MO. Phase transitions and universal dynamics in confined films. Phys. Rev. Lett. 1992; 68: 3448-3451.

[15] Gao J, Luedtke WD, Landman U. Layering transitions and dynamics of confined liquid films. Phys. Rev. Lett. 1997; 79: 705-708.

[16] Demirel AL, Granick S. Glasslike transition of a confined simple fluid. Phys. Rev. Lett. 1996; 77: 2261-2264. 
[17] Gourdon D, Israelachvili JN. Transitions between smooth and complex stick-slip sliding of surfaces. Phys. Rev. 2003; E 68: 21602.

[18] Drummond C, Israelachvili JN. Dynamic phase transitions in confined lubricant fluids under shear. Phys. Rev. 2001; E63: 41506.

[19] Mazuyer D, Cayer-Barrioz J, Tonck A, Jarnias F. Friction dynamics of confined weakly adhering boundary layers. Langmuir 2008; 24: 3857-3866.

[20] Georges JM, Tonck A, Loubet JL, Mazuyer D, Georges E, Sidoroff F. Rheology and friction of compressed polymer layers adsorbed on solid surfaces. J. Phys. 1996; 6: 57-76.

[21] Luengo G, Heuberger M, Israelachvili JN. Tribology of shearing polymer surfaces. Part II - Polymer (PBMA) sliding on Mica. J. Chem. Phys. 2000; 104: 7944-7950.

[22] Cayer-Barrioz J, Mazuyer D, Tonck A, Yamaguchi E. Frictional rheology of a confined adsorbed polymer layer. Langmuir 2009; 25: 10802-10810.

[23] Schallamach A. How does rubber slide. Wear 1971; 17: 301-312.

[24] Dieterich J. Modeling of rock friction 1. Experimental results and constitutive equations. J. Geophys. Res. 1979; B 84: 2161.

[25] Rice JR, Ruina AL. Stability of steady frictional slipping. J. Appl. Mech. 1983; 105: 343-349.

[26] Ruina AL. Slip instability and state variable friction laws. J. Geophys. Res. 1983; B 88: $10359-10370$.

[27] Carlson JM, Batista AA. Constitutive relation for the friction between lubricated surfaces. Phys. Rev. 1996; E 53: 4153-4165.

[28] Persson BNJ. Theory of friction: The role of elasticity in boundary lubrication. Phys. Rev. 1994; B 50: 4771-4786.

[29] Baumberger T, Berthoud P, Caroli C. Physical analysis of the state- and ratedependent friction law. II. Dynamic friction. Phys. Rev. 1999; B 60: 3928-3939.

[30] Drummond C, Israelachvili JN, Richetti P. Friction between two weakly adhering boundary lubricated surfaces in water. Phys. Rev. 2003; E 67: 066110.

[31] Persson BNJ. Theory of friction: Stress domains, relaxation, and creep. Phys. Rev. 1995; B 51: 13568-13585.

[32] Lemaître $A$, Carlson J. Boundary lubrication with a glassy interface. J. Phys. Rev. 2004; E 69: 61611.

[33] Chernyak YB, Leonov AI. On the theory of adhesive friction of elastomers. Wear 1986; 108: 105-138.

[34] Mazuyer D, Tonck A, Cayer-Barrioz J. Friction control at the molecular level: from superlubricity to stick-slip. In: Erdemir A, Martin JM, editors. Superlubricity, Elsevier BV; 2007, p. 397-426.

[35] Johnson KL, Kendall K, Roberts AD. Surface energy and the contact of elastic solids. Proc. R. Soc. Lond. 1971; A324, 1558: 301-313.

[36] Sinou J], Dereure O, Mazet GB, Thouverez F, Jezequel L. Friction-induced vibration for an aircraft brake system - Part 1: Experimental approach and stability analysis. International Journal of Mechanical Sciences 2006; 48: 536-554.

[37] Sinou JJ, Fritz G, Jézéquel L. The role of damping and definition of the Robust Damping Factor (RD-Factor) for a self-exciting mechanism with constant friction. Journal of Vibration and Acoustics 2007; 129: 297-306.

[38] Chevillot F, Sinou J], Hardouin N. Nonlinear transient vibrations and coexistences of multi-instabilities induced by friction in an aircraft braking system. Journal of Sound and Vibration 2009; 328: 555-574.

[39] Chui CK. An Introduction to Wavelets. NewYork: AcademicPress; 1992.

[40] Torrence C, Compo G. A practical guide to wavelet analysis. Bulletin of the American Meteorological Society 1998; 79: 61-78. 